\title{
Air quality health indices - review
}

\author{
Anna Gayer $^{1}$, Lukasz Adamkiewicz ${ }^{2,3,}$, Dominika Mucha $^{2}$, Artur Badyda ${ }^{2}$ \\ ${ }^{1}$ The Main School of Fire Services, Faculty of Fire Safety Engineering, 52/54 Slowackiego St., \\ 01-629 Warsaw, Poland \\ ${ }^{2}$ Warsaw University of Technology, Faculty of Building Services, Hydro and Environmental \\ Engineering, 20 Nowowiejska St., 00-653 Warsaw, Poland \\ ${ }^{3}$ European Clean Air Centre, 10/6 Felicjanek St., 33-332 Cracow, Poland
}

\begin{abstract}
Many studies have shown associations between exposure to air pollutants and negative health effects such as increased number of Hospital Admissions for respiratory and cardiovascular diseases or even increased daily mortality due to those causes. To assess air quality in ambient air continuous monitoring is run in many cities worldwide. Data which is collected at these points should represent exposure of the population and is used to monitor medium and long-term trends. To provide an information for citizens about the impact of air quality on their health several governmental and municipal agencies developed air quality health indices These tools are based on environmental epidemiology models and on-line air quality data. The health risk is assessed differently for each index. In this paper review of Canadian, American, Hong Kong's Air Quality Health Index.
\end{abstract}

\section{Introduction}

Many studies have shown associations between short-term exposure to air pollutants and negative health effects. One of the most reliable and comprehensive reviews of such impacts is presented in HRAPIE report (Health Risks of Air Pollution in Europe): Recommendations for concentration-response functions for cost-benefit analysis of particulate matter, ozone and nitrogen dioxide [1]. HRAPIE analysis presents associations between exposure and health outcomes as the concentration-response curves such as:

- $\mathrm{PM}_{2.5}$, daily mean associated with mortality, hospital admissions due to cardiovascular or respiratory diseases;

- $\mathrm{PM}_{10}$, daily mean associated with incidence of asthma symptoms in asthmatic children;

- $\mathrm{O}_{3}$, daily maximum 8-hour mean associated with mortality, minor restricted activity days, hospital admissions.

Development of air quality monitoring and rising public awareness of the harmful influence of air pollutants lead to the implementation of health protection tools connected to air quality. Air quality health indices are designed to enable people to limit short-term exposure to air pollution by adjusting their activities due to increased levels of pollutants in ambient air. There are generally two types of indices:

\footnotetext{
*Corresponding author: lukasz.adamkiewicz@gmail.com
} 
- the Air Quality Index (AQI) which is calculated by comparing the concentrations of selected air pollutants to their established air quality objectives and criteria, and is based on the concentration of a pollutant that is highest relative to its standard and

- the Air Quality Health Index (AQHI) that is based on the associations between shortterm exposure to air pollutants and negative health effects [2].

\section{Materials and Methods}

There are numerous air quality indices worldwide. Review the research presented in this paper was focused only on those in which health outcomes was the variable to compute the index. To prepare unbiased material a full review of the database has been conducted. PubMed and Scopus repository with the following search formula "air" and "quality" and "health" and "index" in title or abstract was used. This has returned 354 results in PubMed and 42 in Scopus. Next step of the process was to select only those papers that address the issue of ambient air quality and use health as the variable for development of the index. In this process 39 works from PubMed and 13 from Scopus were selected for full text analysis. Only original research articles were included. Another condition to include the paper in this review was a detailed equation explained in the text. The minimum requirement to be included in this review article was to at least use World Health Organization Air Quality Guidelines (WHO AQG) as reference value [3]. The final result showed 10 original air quality health indices which are reviewed in this paper.

\section{Results}

Reviewed articles which present the development of air quality index with the variable of health can be divided into 3 main groups:

- with use of WHO AQG as a threshold for index levels

- with use of an impact on health parameter instead of concentration of air pollutant ie. relative risk, excess risk or sum of those variables;

- produced short-term ambient air pollution impact analysis with the methodology of time series or cross-sectional studies which is then implemented into the equation.

\subsection{Air quality health index based on WHO AQG}

The simplest air quality health index uses a WHO AQG. This approach was done in Hong Kong, where the sum of selected air pollutants: $\mathrm{PM}_{10}, \mathrm{SO}_{2}, \mathrm{NO}_{2}, \mathrm{O}_{3}$ and its $3 \mathrm{~h}$ max value is compared to WHO AQG (1):

$$
s_{i}=100 *\left(\frac{q_{i}}{Q_{i}}\right)
$$

where $s_{i}$ is the value of sub-index of $i$ pollutant, $q_{i}$ is the concentration of $i$ pollutant and $Q_{i}$ is the value of WHO AQG for $i$ pollutant. The sum of sub-indices was calculated to form the value of HKAQI - equation (2).

$$
H K A Q I=\left(\frac{1}{n} * \sum_{i=1}^{n} s_{i}^{p}\right)^{\frac{1}{p}}
$$


where $n$ is the number of pollutants, and $p$ is the number from 1 to infinity. Since the formula uses the sum it is vulnerable to low concentration of 2-3 pollutants, which in result could give a rank of healthy air status. To overcome this problem $p$ variable have been introduced. Researchers have analyzed the historical air pollution monitoring data to determine the value of $p$. They checked what $p$ would result in the lowest over- and underestimate of the HKAQGI taking into account that at least 1 pollutant should have the value over $1.5 \times$ WHO AQG or 2 pollutants $1.0 \times$ WHO AQG. The value of $p$ for Hong Kong equals 3 . The index has 5 categories: low (0-50), moderate (51-100), high (101-150), very high (151-200), serious (above 201) [4].

Another example of WHO AQG impact-related air quality index is called DAQ and was developed for southwestern Germany[5]. It uses short-term air pollution stress developed to produce category thresholds for each pollutant. Index values are calculated based on equation (3):

$$
D A Q x=\left[\left(\frac{D A Q x_{\text {up }}-D A Q x_{\text {low }}}{C_{\text {up }}-C_{\text {low }}}\right) * C_{\text {inst }}-C_{\text {low }}\right]+D A Q x_{\text {low }}
$$

where $D A Q x$ is the value of index, $D A Q x_{u p}$ is the upper value boundary of the index, $D A Q x_{\text {low }}$ is lower value boundary of the index category, $C$ are the upper and lower concentration, that belongs to the index category $C_{\text {inst }}$ is the current concentration of $x$ pollutant. $D A Q$ index have 6 categories depending on $D A Q x$ value that has following ranges $<1.4,1.5-2.4,2.5-3.4,3.5-4.4,4.5-5.4,>5.5$. This index is based on short term health outcomes but it is not based strictly on health impact analysis. Multi Pollutant Index (MPI) is complementary to HKAQI [4]. It isbased on the difference between current air quality and given threshold. If it uses WHO AQG it can be called as health base. Equation (4) describes the calculation process:

$$
M P I=\frac{\mathbf{1}}{n} *\left[\sum_{i=1}^{n}\left(\frac{A C_{i}-G C_{i}}{G C_{i}}\right)\right]
$$

where $A C_{\mathrm{i}}$ concentration of $i$ pollutant, $G C_{i}$ guideline value of $i$ pollutant, and $n$ number of pollutants.

This group of indices is normalized with WHO AQG so they are easily applicable in every country, region or city. However, WHO stands that there is not safe threshold for many pollutants, also the AQG was not set to be the same health risk. Therefore using only WHO AQG as the health variable does not fully present the health perspective.

\subsection{Index based on relative or excess risk}

Relative or excess risk is an epidemiological parameter that measures different health stressors. This approach allows to compare different pollutants with the same increase of health outcomes.

Aggregated index (ARI) is the example of simple formula, that uses relative risk as the variable converting short-term air pollution impacts into the scale of index. Researchers took into account aggregation adverse health effects, which is described by the equation (5) [4].

$$
A R I=\sum_{i=1}^{n} a_{i} * C_{i}
$$


where $a_{i}$ is the conversion factor of $i$ pollutant calculated based on the corresponding relative risk and $C_{i}$ current concentration of this pollutant.

Using national epidemiology studies on ambient air pollution, provides health dimension for development of a complex health based index. Health-risk based Air Quality Index (HAQI) in China was created on the basis of excess risk (ER) [6]. In first step relative risk for $i$ pollutant have been calculated using equation (6):

$$
R R_{i}=\exp \left[\beta_{i}\left(m_{i}-m_{i, 0}\right)\right], m_{i}>m_{i, 0}
$$

where $\beta_{i}$ is the exposure-response coefficient, representing the additional health risk of increase concentration of pollutant $i$. The $\beta_{i}$ values have been derived from Chinese studies of short-term exposure to air pollution and daily mortality. Parameter $m_{i}$ equals concentration of pollutant $i$. The value of $m_{i, 0}$ is the threshold of risk and it comes from Chinese Ambient Air Quality Standard 24-h Grade II. Therefore HAQI assumes, that below the level of $m_{i, 0}$ there are no adverse health effects. From this ER have been calculated using equation (7):

$$
E R_{i}=R R_{i}-1
$$

Sum of ER is calculated from all pollutants, which describe equation (8):

$$
E R_{\text {total }}=\sum_{i=1}^{n}\left(R R_{i}-1\right)
$$

Researches looking from health perspective have taken the assumption that concentration $\left(m_{i}^{*}\right)$ of $i$ pollutant is equal to $E R_{\text {total }}$. This so called equivalent concentration is calculated from equation (9):

$$
m_{i}^{*}=\frac{\ln \left(R R_{i}^{*}\right)}{\beta_{i}}+m_{i, 0}
$$

where $R R_{i}^{*}$ is equivalent relative risk. This procedure makes index depended on health risk instead of using untransformed measured concentration of each pollutant $\left(m_{i}\right)$. Categories of HAQI is calculated with following equation (10):

$$
H A Q I=\max \left(A Q I_{i, j} \frac{m_{i}^{*}}{m_{i, j}}\right), j=1,2, \ldots, 6
$$

where AQI is baseline derived from Air Quality Index issued by the Chinese Ministry of Environmental Protection, $m_{i, j}$ is upper limit of $i$ pollutant for concentration for $j$ category. The HAQI uses AQI categories which are: excellent, satisfactory (0-50); good, acceptable (51-100); light pollution, unhealthy for sensitive people (101-150); moderate pollution, unhealthy (151-200); serious pollution, very unhealthy (201-300); very severe pollution, hazardous (above 301). This index is based on ER, which is derived from local epidemiological studies. Categories of HAQI are created from equivalent concentration that is equal to total ER, which means, that it represents the risk of the population of adverse health effects.

Approach based on excess or relative risk allows to normalized the different pollutant from the health perspective. This can be done using international or regional epidemiological study. Local ones give better perspective but can be used only in the place where the health outcomes were derived from ambient air quality. 


\subsection{Index based on epidemiological studies}

The most complex procedure to develop index requires exposure - response coefficient from time series, cross-sectional studies or other epidemiological analysis. Results give a value, that represents the number of daily excess death, that can be linked to a particular air pollutant. This is the most complex procedure in developing an air quality health index since it requires a to process a large data set.

The first index worldwide, that implemented this approach has been introduced in Canada and it is called Air Quality Health Index (AQHI) [7]. It was constructed from the sum of excess mortality risk associated with individual pollutants from a time-series analysis of air pollution and mortality in Canadian cities and adjusted to a $0-10$ scale. It is calculated hourly on the basis of trailing $3-\mathrm{hr}$ average pollutant concentrations. The concentration response function was determined using time series, taking into account daily mortality and 6 pollutants $\left(\mathrm{CO}, \mathrm{NO}_{2}, \mathrm{O}_{3}, \mathrm{PM}_{10}, \mathrm{PM}_{2.5}, \mathrm{SO}_{2}\right)$. All concentrations were $3 \mathrm{~h}$ average except $\mathrm{PM}_{10}$ and $\mathrm{PM}_{2.5}$, that was $24 \mathrm{~h}$ and in later process recalculated to $3 \mathrm{~h}$ max values. The analysis was done using Generalized Additive Model with quasi-Poisson distribution. Next step was taking into account different number of population in the cities and levels of exposure to pollution, it was done according to equation (11):

$$
M W E D=\sum_{j=\mathbf{1}}^{n}\left[\left(\frac{m_{j}}{\sum_{j=\mathbf{1}}^{n} m_{j}}\right) * \sum_{i=\mathbf{1}}^{p} 100 *\left(\exp ^{\beta_{i} * x_{i, j, t}-1}\right)\right]
$$

where $M W E D$ is mortality weighted excess deaths (\%), $\beta_{i}$ is the $i$ regression coefficient from model, that links $i$ pollutant with mortality, $x_{i, j, t}$ is the concentration of $i$ pollutant in the $j$ city at the $t$ hour corresponding to maximum daily excess mortality, $n$ is the number of cities and $m_{j}$ is the daily average number of deaths in $j$ city. Next step was to get the maximum number of deaths attributable to air pollution to make a 10 value AQHI, which is described in equation (12):

$$
c=\max _{t=1 \ldots q}\left\{\sum_{j=\mathbf{1}}^{n}\left[\left(\frac{m_{j}}{\sum_{j=\mathbf{1}}^{n} m_{j}}\right) * \sum_{i=\mathbf{1}}^{p} 100 *\left(\exp ^{\left.\beta_{i} * x_{i, j, t}-1\right)}\right]\right\}\right.
$$

where $q$ is number of days, and $c$ is the maximum mortality weighted excess deaths. From that, the general index formula is design, and presented in equation (13):

$$
A Q H I=\frac{10}{c} \sum_{i=1}^{p} 100 *\left(\exp ^{\beta_{i} * x_{i}}-1\right)
$$

where $i$ is the regression coefficient, that links $i$ pollutant with mortality, $p$ is the number of pollutants, and $c$ is the scaling factor, which is the maximum mortality weighted excess deaths. Researches checked different sets of pollutants to fit the best scales for cities and also checked the seasonal difference of index (warm and cold). The best fit for whole year, have 3 pollutants $-\mathrm{NO}_{2}, \mathrm{O}_{3}, \mathrm{PM}_{2.5}$ (instead of $\mathrm{PM}_{10}$ ) and is described by formula (14):

$$
P M_{2.5} A Q H I=\frac{10}{10.4} *\left[100 *\left(\exp ^{0.000871 * N O_{2}}-1+\exp ^{0.000537 * O_{3}}-1+\exp ^{0.000487 * P M_{2.5}}\right)\right]
$$


This work have utilized the time series analysis, different set of pollutant, provided best fit for different studies and allowed for other researchers around the world to copy the concept and made a tailored solution for particular regions, cities.

There are other indices that uses the same approach. Air Quality Health Index (AQHI) developed in China is based on mortality - pollution association [8]. AQHI is calculated from the following equation (15):

$$
A Q H I_{t}=10 * \text { daily total } E R_{t} / \max
$$

where daily total $E R_{t}$ stands for the sum of $E R_{i t}$ of mortality associated with the $i$ pollutant on $t$ day. Categories of AQHI have been selected on the base of WHO AQG.

Another Chinese index is based on excess risk but with a different end point [9]. It takes into account daily HAs for respiratory and CADs associated with four air pollutants. It can be described with the following equation (16):

$$
\text { \%ER }=\sum_{i=1, \ldots, p}\left(\exp ^{\beta_{i} x_{i, j}-\mathbf{1}}\right) * \mathbf{1 0 0 \%}
$$

where $\beta_{i}$ was the regression coefficient of pollutant $\mathrm{i}$ from the time series analysis, and $x_{i j}$ was the concentration of pollutant $i$ at time $j$, and $p$ number of total pollutants. The $\% \mathrm{ER}$ was calculated using the concentrations of four pollutants $-\mathrm{NO}_{2}, \mathrm{O}_{3}, \mathrm{PM}_{10}$ and $\mathrm{SO}_{2}$, for each day. Researchers also calculated ER for vulnerable groups like children under age 5 and elderly people over 65. Categories of the AQHI was calculated using WHO AQG for short-term exposure, then \%ER was sum from 4 pollutants which gave the upper bound of the index. Other bands of index were developed in relation to this highest limit formed 5 category and 10 band index: low (1-3 cat., $\% E R>0-5.64)$; moderate (4-6 cat., $\% E R>5.64-$ 11.29); high ( 7 cat., $\% E R>11.29-12.91$ ); very high (8-10 cat., $\% E R>12.91-19.37$ ); serious (higher than 10 cat. $\% E R>19.37$ ). This index uses health variable, that is based on local times series studies, and take into account other effects than mortality, which for the shortterm informing public is very important.

The third approach to introduce Air Quality Health Index based Canadian AQHI in China was done in Shanghai. [10]. Short-term mortality as the end point and formulated bipollutant index instead of multi one was included. Researchers provided 2 formulas (17) and (18) depending what fraction of PM is monitored:

$$
\begin{aligned}
& P M_{10} A Q H I=\frac{10}{17} * 100 *\left[\exp ^{\left.0.000154 * P M_{10}-1+\exp ^{0.000664 * N O_{2}}-1\right]}\right. \\
& P M_{2.5} A Q H I=\frac{10}{15} * 100 *\left[\exp ^{\left.0.000172 * P M_{10}-1+\exp ^{0.000664 * N O_{2}}-1\right]}\right.
\end{aligned}
$$

where, $\mathrm{PM}_{10} \mathrm{AQHI}$ and $\mathrm{PM}_{2.5} \mathrm{AQHI}$ are the values of index created on the base of the appropriate PM fraction, arguments of exp are the value of increase of total mortality with $10 \mu \mathrm{g} / \mathrm{m}^{3}$ increase of daily concentration of appropriate pollutant (regression coefficients). Number 17 and 15 are the scaling values representing the maximum number of daily excess deaths associated with $\mathrm{PM}_{10}$ and $\mathrm{PM}_{2.5}$ concentration. Proposed formula uses well recognized Canadian AQHI, difference is in the number of pollutant, where original conception added also $\mathrm{O}_{3}$ to equation also Chinese used mortality as the health end point. Introduction of such indexes for particular cities and regions requires epidemiological studies. Health data for these research must be disaggregated in order to identify the daily effects of air pollution. In addition, analysis of these types requires a number of confounding variable that are spatially distributed. 


\subsection{Other health-related ambient air quality indices}

A different approach apart from those explained above has been used by researchers, who have chosen a fuzzy-analytical hierarchical process to determine a Fuzzy Air Quality Health Index (FAQHI) [11]. Hierarchy tree (part of fuzzy analytical) has been build where $2^{\text {nd }}$ layers are called: Pollutant Index consisted of concentration $\left(\mathrm{PM}_{10}, \mathrm{SO}_{2}, \mathrm{NO}_{2}, \mathrm{CO}, \mathrm{O}_{3}\right)$ and Exposure Index which consist of location sensitivity, population sensitivity and population density. Pollutant Index has a weight of 0.74 and Exposure Index 0.26. In the last step of creating the index the defuzzification of FAQHI was done, that describes equation (19):

$$
F A Q H I=1 * r^{l}+2 * r^{M}+3 r^{H}
$$

where, fuzzy sets are expressed by low $\left(r^{L}\right)$, medium $\left(r^{M}\right)$ and high $\left(r^{H}\right)$ and those values were derived as represented in matrix $u$, number 1,2,3 are weights for non-fuzzy value. This approach allows to implement the issued related to public health as population sensitivity and density. However it does not put the emphasis on each pollutant, that has another impact on health, therefore FAQHI omits the importance of looking into air pollutants from the health perspective.

Very well recognized AQHI can be added with a new variable. Researchers have developed Air Quality Risk Index (AQRI) [12]. It is based on standard AQHI approach with the addition of safety risk (damage and loses) and environment. It is described by equation (20):

$$
A Q R I=\frac{R_{H} * W_{H}+R_{S} * W_{S}+R_{E} * W_{E}}{W_{H}+W_{S} * W_{E}}
$$

where, $A Q R I$ is Air Quality Risk Index, $R_{H}$ is a health risk, $\mathrm{R}_{\mathrm{S}}$, is a safety risk, $\mathrm{R}_{\mathrm{E}}$, is environment risk, $W$ are corresponding weighting factors, that each value is higher than 0 and lower than 1.

Last presented air quality health index, that has been investigated was very specific since it was designed for tire fire air pollution. It was created due to 18 days fire of tires in a landfill [13]. Researchers wanted to include the hazardous compounds, that being produced during the fire. The index is based on two reference groups of concentration of pollutants National Ambient Air Quality Standards (NAAQS) from Environmental Protection Agency of USA and Acute Exposure Guideline Levels (AEGL-1). If there is no reference of substance in NAAQS, researches looked into AEGL-1 with $1 \mathrm{~h}$ concentration that is defined as the 'airborne concentration of a substance above which it is predicted that the general population, including susceptible individuals, could experience notable discomfort, irritation, or certain asymptomatic non sensory effects'. Therefore researches included substances, that is not measured by national monitoring service but are harmful to human health. Equation (21) explains how the AQI for burning tires are calculated:

$$
a_{\text {tot }}=\left(a_{P M}^{p}+o_{1}^{p}+o_{2}^{p}+\cdots+o_{m}^{p}+u_{1}^{p}+u_{2}^{p}+\cdots+u_{n}^{p}\right)^{\frac{1}{p}}
$$

where $a_{P M}$ is concentration of $\mathrm{PM}_{2.5}, o_{m}$ are co-pollutants, and $u_{n}$ represents unmeasured compounds, $p$ is the value that controls the nature of the summation process. Researchers have conducted that value of 1 for $p$ fits the best for this purpose. This index takes into account compounds, that are not measured in the standard ambient monitoring network. However, it does not allow its use in normal conditions since additional measurement which can be done during fires are needed. 


\section{Conclusions}

Search for articles describing the development of air pollution health index has shown that only a few papers were yet published in this area. From total 396 papers, only 10 of them include health as a variable for computing the air quality index. Review presents 3 main approaches which are used to create air quality health indices. Authors of those use either WHO AQG as a threshold for index levels or parameters which reflects how current air quality influences human health (ie. relative risk, excess risk or sum of those variables) or the results of short-term effects analysis.

The biggest disadvantage of air quality health indices is their local utility. A study conducted in Canada showed that there are many differences between each city. Creating air quality health index on the continent level like Europe can lead to false results, since there is a difference in the quality of life in different countries that would act as confounding factor of the statistical quality of the index. Simplest utilization of WHO AQG or related to relative risks is possible across different countries since those values are constant. From the Polish perspective, it seems, that there is a need for epidemiolocal evidence based on long cohort studies, which could allow development of exposureresponse coefficient values for Polish air quality health index.

\section{References}

1. World Health Organization, Health risks of air pollution in Europe - HRAPIE project Recommendations for concentration-response functions for cost-benefit analysis of particulate matter, ozone and nitrogen dioxide, (2013)

2. H. Chen, R. Copes, Environmental and Occupational Health (Queen's Printer for Ontario, 2013)

3. World Health Organization, Air Quality Guidelines Global Update 2005: Particulate Matter, Ozone, Nitrogen Dioxide and Sulfur Dioxide (Copenhagen World Health Organization Regional Office for Europe, 2006)

4. T.Q. Thach, H. Tsang, P. Cao, L.M. Ho, Int. J. Hyg. Environ. Health 221(1), 17 (2018)

5. H.L. Lokys, J. Junk, A. Krein, Int. J. Environ. Health Res. 25(1), 52 (2015)

6. F. Shen, X. Ge, J. Hu, D. Nie, L. Tian, M. Chen, Environ. Res. 156, 625 (2017)

7. D.M. Stieb, R.T. Burnett, M. Smith-Doiron, O. Brion, H.H. Shin, V. Economou, J. Air Waste Manag. Assoc. 58(3), 435 (2008)

8. J. Hu, Q. Ying, Y. Wang, H. Zhang, Environ. Int. 85, 17 (2015)

9. T.W. Wong, W.W.S. Tam, I.T.S. Yu, A.K.H. Lau, S.W. Pang, A.H.S. Wong, Atmos. Environ. 76, 52 (2013)

10. R. Chen, X. Wang, X. Meng, J. Hua , Z. Zhou, B. Chen, H. Kan, Environ. Int. 51, 168 (2013)

11. A.K. Gorai, T. Kanchan, A. Upadhyay, F. Tuluri, P. Goyal, P.B. Tchounwou, Sci. Total Environ. 15:533, 495 (2015)

12. A. Ahmadi, M. Abbaspour, R. Arjmandi, Z. Abedi, Int. J. Environ. Sci. Technol. 12(12), 3773 (2015)

13. A. Singh, S.N. Spak, E.A. Stone, J. Downard, R.L. Bullard, M. Pooley, P.A. Kostle, M.W. Mainprize, M.D. Wichman, T.M. Peters, D. Beardsley, C.O. Stanier, Atmos. Environ. 104, 273 (2015) 